\title{
The CDMS II Experiment: Dark Matter results from the complete data set
}

\author{
Tarek SAAB for the CDMS II Collaboration* \\ University of Florida \\ E-mail: tsaabaufl. edu
}

\begin{abstract}
Over the past decade, the Cryogenic Dark Matter Search (CDMS II) experiment has provided world-leading sensitivity for the direct detection of Weakly Interacting Massive Particle (WIMP) dark matter. This presentation will discuss the results from the analysis of the complete CDMS II Ge data set in which two nuclear-recoil events where seen with an expected background of $0.9 \pm$ 0.2 events. Although this does not constitute statistically significant evidence for a WIMP signal the CDMS II data is able to place strong constraints on the WIMP-nucleon spin-independent scattering cross-section for a wide range of WIMP masses, excluded new parameter space in inelastic dark matter models as well as exclude parameter space for for other hypothesized dark matter models. The present status and future plans for the followup SuperCDMS experiment will also be discussed.
\end{abstract}

Identification of Dark Matter 2010

July 26 - 302010

University of Montpellier 2, Montpellier, France

\footnotetext{
* Speaker.
} 


\section{The CDMS II Experiment}

The Cryogenic Dark Matter Search (CDMS) II experiment is designed to detect evidence of non-baryonic dark matter $[1,2]$ via the direct interaction of the dark matter particles in the galactic halo with detector nuclei. Under the assumption that the properties if dark matter particles are described by supersymmetric extensions to the Standard Model (SUSY) as a generic class of particles having a mass in range of few $\mathrm{GeV} / \mathrm{c}^{2}--\mathrm{TeV} / \mathrm{c}^{2}$ and a very low scattering cross-section with Standard Model (WIMPs) [3] their recoil energy spectrum with terrestrial detectors is given by Equation $1.1[3,4]$ :

$$
\frac{d R}{d Q}=\frac{\sigma_{0} \rho_{0}}{\sqrt{\pi} v_{0} m_{\chi} m_{r}^{2}} F^{2}(Q) T(Q)
$$

where $\rho_{0}$ is the WIMP density in the local neighborhood within the galactic halo, $\sigma_{0}$ is the elastic scattering cross-section between the WIMP and nucleus, $m_{r}$ is the WIMP-nucleus reduced mass: $m_{r}=\left(\frac{m_{\chi} m_{N}}{m_{\chi}+m_{N}}\right), F(Q)$ is the nuclear form factor, and $T(Q)$ is the integral of WIMP local velocity distribution. For spin-independent scattering, $\sigma_{0}$ is approximately proportional to the WIMPproton scattering cross-section $\sigma_{\chi-p}: \sigma_{0} \propto A^{2} \sigma_{\chi-p}$. This relationship emphasizes the dependence of the interaction rate on the size of the nucleus $A$.

The resulting spectrum is roughly exponential, with a mean recoil energy in the tens of $\mathrm{keV}$ and event rates of less than $10^{-4}$ events $/ \mathrm{kg} / \mathrm{keV} /$ day. The expected signal rate is orders orders of magnitude lower than the background rates achieved in the cleanest detector materials, typically $\leq 1$ event $/ \mathrm{kg} / \mathrm{keV} /$ day, therefore the ability to distinguish potential signal events from background interactions is essential for the success of a direct detection experiment.

\subsection{Background Discrimination}

The CDMS II experiment uses semiconducting Ge as well as Si crystals as the dark matter detectors [5]. A fraction of the energy deposited by an interaction results in the creation of electronhole pairs, with the remainder of the energy creating a population of high frequency athermal phonons. We apply an electric field of $3 \mathrm{~V} / \mathrm{cm}$ across the crystal to allow the electrons and holes to drift to opposing electrodes, where they are subsequently measured with a charge amplifier. The energy deposited in the phonon system is measured by four Quasiparticle-assisted ElectrothermalFeedback Transition-Edge-Sensors (QETs) that are photolithographically patterned on the surface into independent quadrants $[6,7]$. In the $\mathrm{keV}$ momentum transfer range WIMPs interact with atomic nuclei (referred to as nuclear-recoils), whereas the majority of backgrounds are due to electromagnetic interactions with the atomic electrons (referred to as electron-recoils). For a given amount of imparted energy, a recoiling nucleus travels a much smaller distance in the detector than a recoiling electron resulting in a larger local deposited energy density and suppressed electron-hole generation compared to a recoiling electron. The ratio of the ionization to phonon signals (referred to as ionization yield) permits the rejection of electron recoils [8] with an efficiency of better than 1 in $10^{4}$ [5]. Events occurring within $\sim 10 \mu \mathrm{m}$ of the detector surfaces, have a diminished ionization response resulting in a degradation of the electron-recoil identification to $99.79 \%$. The information in the time structure of the phonon pulse, however, can be used to identify events occurring near the surface versus those occurring in the bulk, and thus improve the surface electron rejection, resulting in an overall electron recoil rejection of better than 1 in $10^{6}$. 


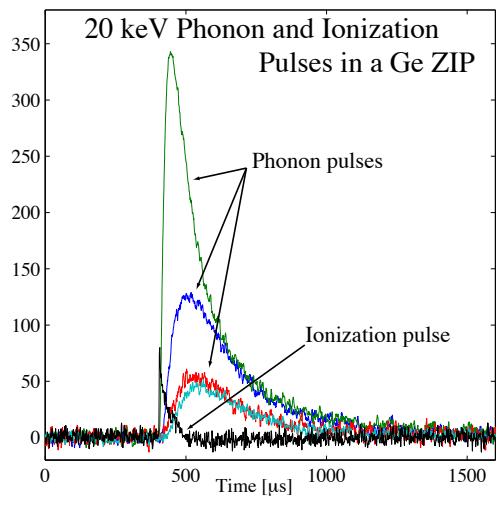

(a)

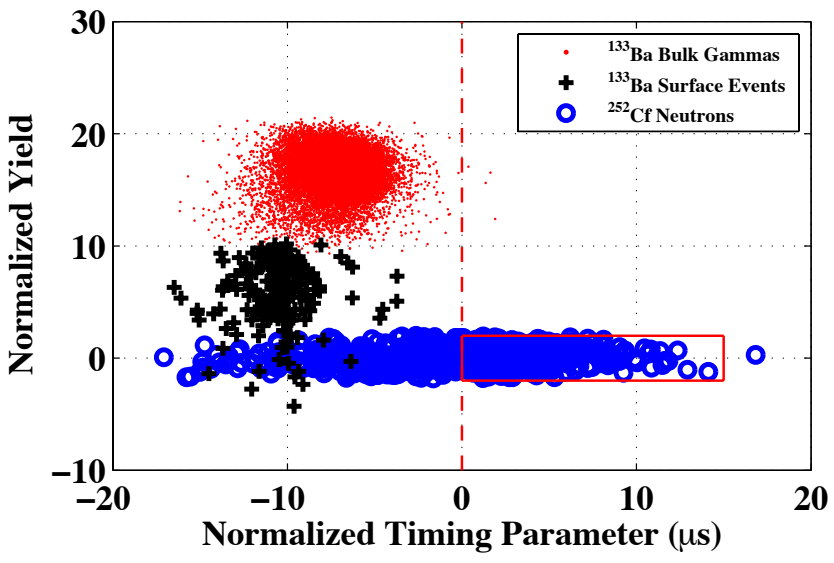

(b)

Figure 1: (a) Phonon (colored) and ionization (black) pulses from a $20 \mathrm{keV}$ event in a Ge detector. The variation in pulse height and timing allows the determination of an interactionÕs position. The rise-time $\mathrm{f}$ the largest phonon pulse, and its delay relative to the prompt ionization pulse are used in defining the timing parameter used in rejecting surface events. (b) Ionization yield vs timing parameter (see text) for calibration data in a Ge detector. The population of events with normalized yield of $\sim 12-20$ and low timing parameter (dots) corresponds to bulk electron recoils. Low yield-low-timing parameters events $(+)$ correspond to surface electron recoils. The population with normalized yield $\sim 0$ and a wide timing-parameter distribution corresponds to bulk nuclear recoil events (o). The vertical dashed line indicates the minimum timing parameter allowed for candidate dark matter events in this detector, and the box shows the approximate signal region.

\section{Results of the CDMS II Nuclear Recoil Analysis}

The data presented here is from a data set taken between July 2007 and September 2008. Due to their larger sensitivity to spin-independent WIMP scattering, only the $19 \mathrm{Ge}$ detectors were included in this WIMP search analysis. The exposure used for this analysis totaled $612 \mathrm{~kg}$-days, after removing periods of poor detector performance [9]. Signal candidates were defined as those events that pass the following criteria:

- A recoil energy between $10 \mathrm{keV}$ and $100 \mathrm{keV}$ in a single detector.

- Lie between $2 \sigma$ of the mean ionization yield of nuclear recoils and more than $3 \sigma$ away from the mean ionization yield of electron recoils.

- Have an ionization signal larger than $4.5 \sigma$ of the noise level of the detector in which it occurred.

- Occur within a fiducial volume defined by the ionization electrodes.

- Have phonon timing characteristics of a nuclear recoil.

- No identifiable energy deposition in the rest of the detector array or in the scintillator shield.

- Satisfy all data quality criteria 
The determination of the above-mentioned cuts was done in a "blind" fashion in which the exact definition of the cuts were made based on an event set which excluded the signal region and its vicinity in parameter space. A sample of calibration events from a ${ }^{252} \mathrm{Cf},{ }^{133} \mathrm{Ba}$ sources as well as multiply-scattered events were used to determine the fraction of nuclear recoil events passing the signal cuts as well as the fraction of misidentified background events. For the total exposure of this analysis $0.8 \pm 0.1$ (statistial) \pm 0.2 (systematic) events were expected to be misidentified as WIMP candidates. The cosmologically produced neutron rate was determined to be $\sim 0.1$ events, which would be indistinguishable from a WIMP signal.

Two events were observed to pass all the signal selection cuts. The events occurred in different detectors and were separated in by several months. Their position in the normalized yield vs. timing parameter space is shown in Figure 2(a). Based on the exposure and the background leakage estimates, the probability of observing two or more misidentified background events is $23 \%$. In order to determine the "robustness" of the two observed events we varied the threshold of the timing cut between the strict and relaxed extremes. Tightening the timing cut such that the expected misidentified background events is reduced to 0.4 would also eliminate both observed events while reducing the WIMP exposure by $28 \%$. Additional events would pass the cuts if it is relaxed such that the expected misidentified background events is increased to 1.7 events. This is indicate in Figure 2(c) where the experimental and expected upper limits are calculated as a function of the the timing cut. It can be seen that over the range of cut values the observed limit does not exceed that the expected one by more than $15 \%$.

The WIMP-nucleon spin-independent scattering cross section based on this analysis is constrained to be $<7.0 \times 10^{-44} \mathrm{~cm}^{2}$ and is shown in Figure 2(b). The cross section upper limit becomes $<3.8 \times 10^{-44} \mathrm{~cm}^{2}$ when these results are considered in combination with the previous CDMS II data sets. An analysis of the data from the point of view of inelastic WIMP-nucleon scattering is presented in elsewhere in these proceedings (see Arrenberg et al).

\section{The Electron Recoil Analysis}

We also searched the low energy electron-recoil spectrum for evidence of a peak due to mono-energetic electron-recoil interactions within the detectors. An exposure of $443.2 \mathrm{~kg}$-days is considered and the same signal selection criteria as described in the previous section were applied, with the exception of the yield and timing cuts. The observed background rate was $\sim 1.5$ events $/ \mathrm{kg} / \mathrm{day} / \mathrm{keV}$ with peaks at $6.54,8.98$, and $10.36 \mathrm{keV}$ as shown in Figure 3(a). The 3 peaks originate from the products of $\mathrm{Ge}$ interactions with cosmic rays (during detector production) and neutrons (during calibration periods) [10]. A profile likelihood analysis was performed to search for any excess signal above the background, with no statistically significant excess observed. The 90\% confidence level (CL) upper limit on the total counting rate is shown in Figure 3(b).

The electron-recoil data can also be used to search for evidence of conversion of axions originating in the sun into $\mathrm{keV}$ scale $\mathrm{x}$-rays. The axion-photon coupling to the nuclear Coulomb field in the detectors converts axions into photons of the same energy (Primakov effect). The conversion probability is strongly correlated with the relative direction between the incident axions beam and the detector's crystal planes. This provides a temporally variable signature of the axion signal as the detector axes revolve with a period of 24 hours. The event rate, as a function of energy and the 


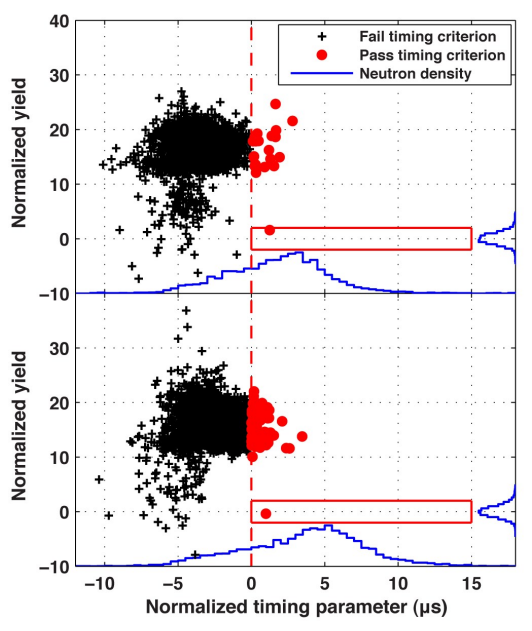

(a)

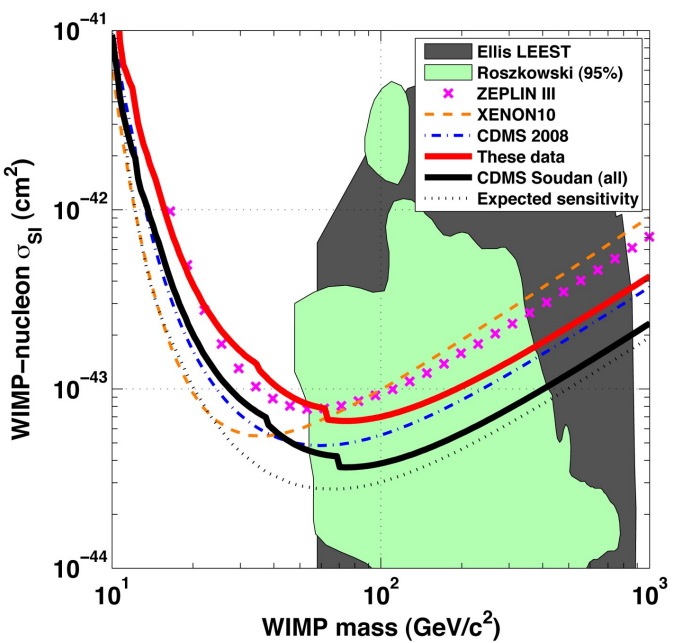

(b)

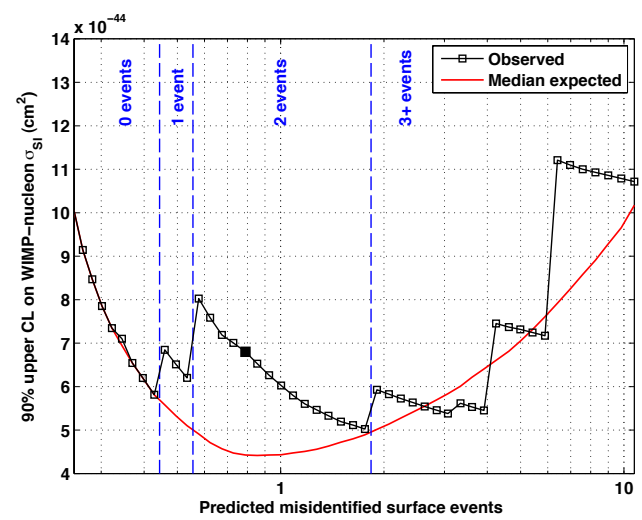

(c)

Figure 2: (a) Normalized ionization yield versus normalized timing parameter for events, in two detectors (T1Z5 and T3Z4), passing all signal selection cuts with the exception of the yield and timing cuts. The signal selection region is indicated by the solid red boxes and contain the two observed events [9]. (b) The observed 90\% C.L. upper limit, at a WIMP mass of $70 \mathrm{GeV} / \mathrm{c} 2$, as a function of predicted misidentified surface event background. The squares show the limits that would have been observed for a given choice of timing cut value. The blue dashed lines mark the transitions at which additional background events would appear. The red curve represents the expected limit corresponding to value of the misidentified events. (c) 90\% confidence upper limits and theoretical allowed regions for the WIMP-nucleon spin-independent cross section as a function of WIMP mass. The red (upper) solid line shows the limit obtained from this analysis and the solid black line shows the combined limit for the full CDMS II data sets. The dotted line indicates the expected sensitivity for this exposure based.

orientation of the crystal relative to the location of the Sun is given by Equation 3.1

$$
R(E)=2 c \int \frac{d^{3} q}{q^{2}} \frac{d \Phi_{a}}{E_{a}}\left[\frac{g_{a \gamma \gamma}^{2}}{16 \pi^{2}}|F(\vec{q})|^{2} \sin ^{2}(2 \theta)\right] W
$$

where $W$ is a detector energy resolution function, $F(\vec{q})$ is the Fourier transform of the electric field 


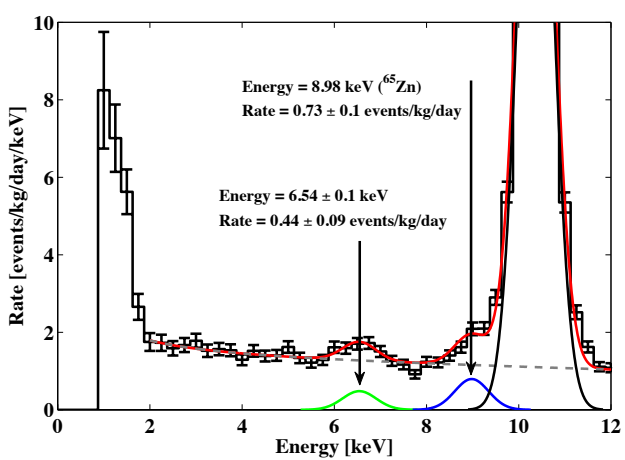

(a)

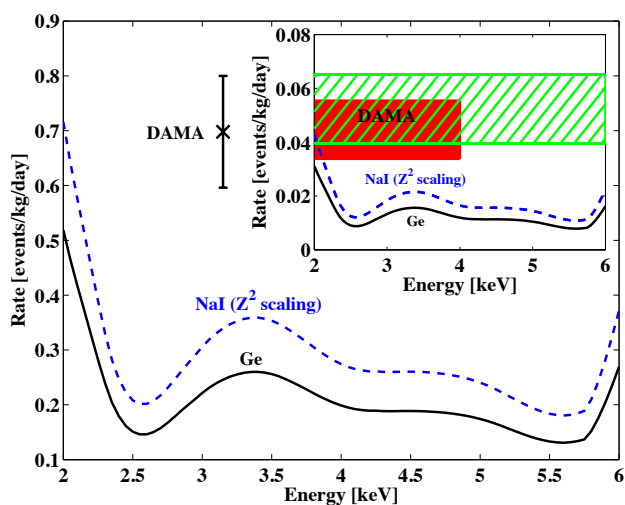

(b)

Figure 3: (a) The measured low-energy spectrum and a background model which includes three Gaussian distribution functions describing the $10.36 \mathrm{keV}$ line from ${ }^{71} \mathrm{Ge}$ (black), the $8.98 \mathrm{keV}$ line from ${ }^{65} \mathrm{Zn}$ (blue) and the $6.54 \mathrm{keV}$ line at the energy of ${ }^{55} \mathrm{Mn}$ (green). (b) $90 \%$ CL upper limit on the total rate in Ge from this analysis (black). The corresponding upper limit on the total counting rate in NaI under the assumption of a $Z^{2}$ scaling of the conversion cross section is shown (blue) for comparison [10].

in the crystal, and $\frac{d \Phi_{a}}{E_{a}}$ is the axion flux at Earth and is given by

$$
\frac{d \Phi_{a}}{E_{a}}=\frac{6.02 \times 10^{14}}{c m^{2} s k e V}\left(\frac{g_{a \gamma \gamma} \times 10^{8}}{G e V^{-1}}\right)^{2} E_{a}^{2.481} e^{-E_{a} / 1.205}
$$

The orientation of the CDMS detector crystal plane was determined up to an uncertainty of $3^{\circ}$ for the azimuthal angle and $<1^{\circ}$ for the zenith angle [11]. For an assumed coupling of $g_{a \gamma \gamma}=$ $10^{-8} \mathrm{GeV}^{-1}$ the time and energy dependent is shown in Figure 4(a). A profile likelihood analysis

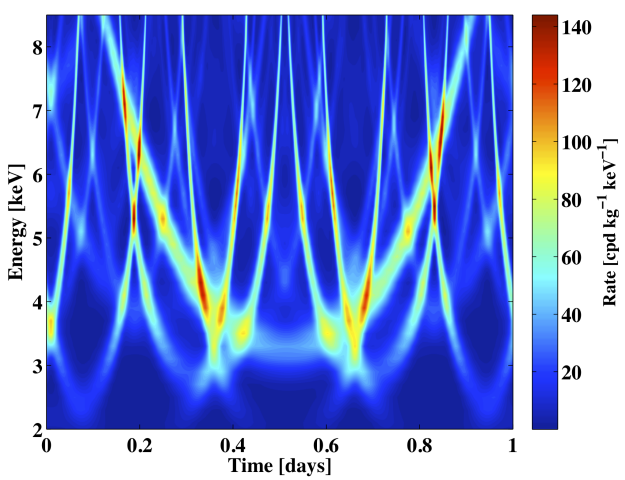

(a)

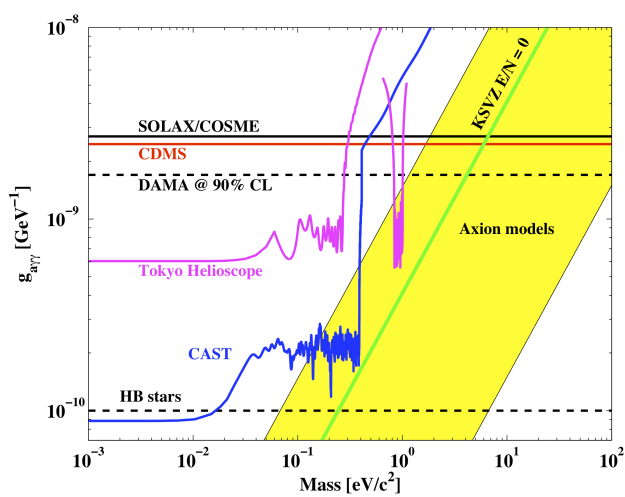

(b)

Figure 4: (a) Time and energy dependence of the expected solar axion conversion rate in a Ge detector for $g_{a \gamma \gamma}=10^{-8} \mathrm{GeV}^{-1}$. (b) The 95\% C.L. upper limit on $g_{a \gamma \gamma}$ from this analysis (red solid line) along with limits from other crystal search experiments (SOLAX and COSME black solid line) and DAMA (upper black dashed line) ) and Tokyo (magenta solid line) and CAST (blue solid line) helioscopes [11].

was performed to determine the best fit value of $g_{a \gamma \gamma}$. The signal rate was determine to be consistent with zero and the $95 \%$ C.L. on $g_{a \gamma \gamma}$ was determined to be $g_{a \gamma \gamma}<2.4 \times 10^{-9} \mathrm{GeV}^{-1}$ as shown in Figure 4(b). 


\section{The SuperCDMS Experiment}

With the data collection portion of the CDMS II experiment at an end, the CDMS collaboration is proceeding with the construction and operation of the SuperCDMS experiment at Soudan. The primary improvements implemented in SuperCDMS are larger detectors: 1 inch thick crystals rather than $1 \mathrm{~cm}$, and an improved sensor design which will enhance the ability of the detector to reject the primary source of misidentified events, namely surface electron-recoils. Increasing the thickness of the detector has the benefit of decreasing the surface electron-recoil background rate due to the reduced surface-to-volume ratio, as well as increasing the production yield, namely more mass per fabrication effort.

The improved sensor design includes two designs referred to as mZIP and iZIP. While the mZIP design is fundamentally similar to the CDMS II design the QET design was changed to increase sensitivity to the timing information in the phonon channel. The layout of the 4 channels was also changed from a quadrant design to one with 3 central channels and an out ring channel as shown in Figure 4. The outer ring channel purpose to better identify event occurring near the outer edge of the detector where the the detector response is not as uniform as that in the central volume. The performance of this detector design was able to achieve a $0.3 \%$ surface electron-recoil misidentification rate with a $77.5 \%$ signal efficiency, which is better than the requirement needed to achieve the SuperCDMS goals [12].
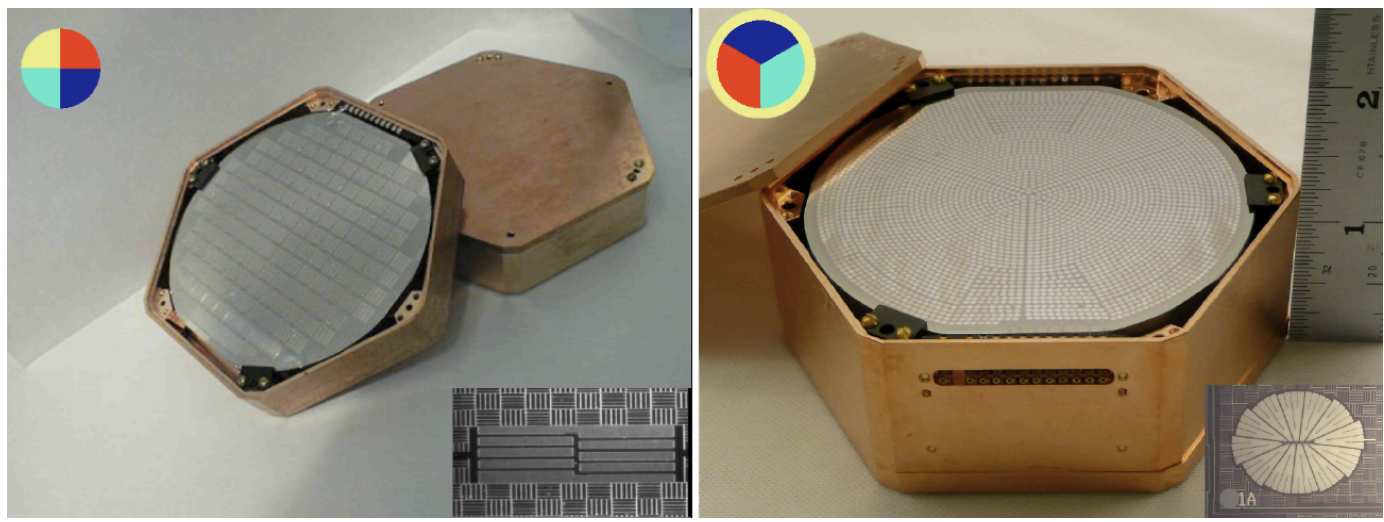

Figure 5: Photographs of a CDMS II detector (left) and a SuperCDMS detector (right). The top left insets show a schematic of the respective phonon sensor configuration. The bottom right insets show a photograph of the QETs.

The iZIP design departs significantly from the the CDMS II detector. The detector has 2 phonon sensors on each face of the detector. The sensors are semi-circular in shape and the diagonal edge of the sensors on one face is rotated by $90^{\circ}$ from the other face. This layout maintains the $\mathrm{x}-\mathrm{y}$ (in the plane parallel to the detector faces) position sensitivity. The depth of an interaction (z-coordinate) is determined by timing and energy differences in the phonon sensors on the two faces. The ionization signal electrodes are interleaved with the phonon sensors on both faces of the detector with a positive voltage bias $(+2 \mathrm{~V})$ applied to the electrodes on one side, a negative bias $(-2 \mathrm{~V})$ applied to the opposite side, and the phonon sensors acting as ground. For events occurring in the bulk of the crystal, the electrons and holes travel to opposite faces of the detector and produce a symmetric signal on the two electrodes. The electrons and holes generated by events 
occurring near the surface of the detector, however, travel to the charged and ground electrode on the same side of the detector producing a signal in only one ionization channel [13]. The sensor layout and electric field configuration is shown in Figure 4. Preliminary tests have shown that the

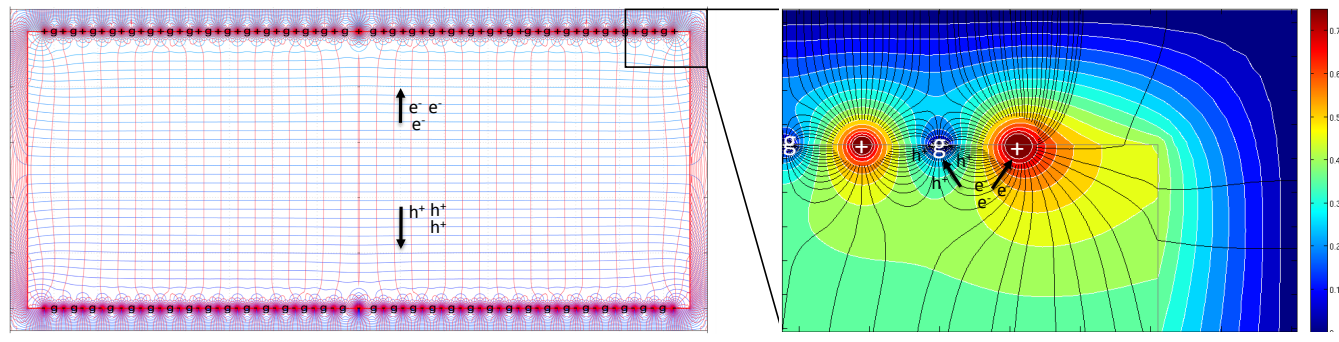

(a)

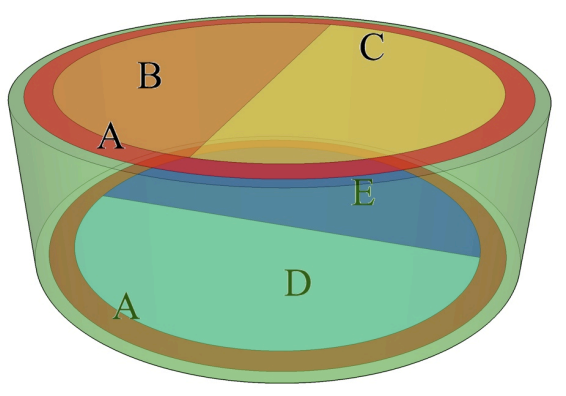

(b)

Figure 6: (a) Left panel: Finite Element Model calculation of electric field lines and equipotential surfaces for an electrode bias of $+2 \mathrm{~V}(-2 \mathrm{~V})$ applied to top (bottom). Right panel: Magnification of the region around the top right corner showing the electrons and holes of a surface event being directed to the same surface. (b) Schematic of the iZIP phonon channel geometry.

performance of the detector in terms surface background misidentification is better than 1 in 3000 based on the yield discrimination alone while maintaining a 69\% signal efficiency. Ionization signal asymmetry discrimination was better than 1 in 1000 with a 59\% signal efficiency. Using timing information from the phonon channels only, a background misidentification of better than 1 in 3000 is achieved with a $20 \%$ loss of signal efficiency. Although it is unlikely that the 3 discrimination methods are uncorrelated, the performance of the iZIP detector easily meets the requirements of the SuperCDMS experiment.

\section{Conclusion}

The completion of the CDMS II data acquisition phase and the beginning of the SuperCDMS experiment are two major milestones in the evolution of the direct detection experiment. With the demonstrated progress of 1 inch iZIP detectors we expect this experiment to continue producing timely and interesting results in the field of dark matter direct detection.

\section{Acknowledgments}

The CDMS collaboration gratefully acknowledges Patrizia Meunier, Daniel Callahan, Pat Cas- 
tle, Dave Hale, Susanne Kyre, Bruce Lambin and Wayne Johnson for their contributions. This work is supported in part by the National Science Foundation (Grant Nos. AST-9978911, PHY-0542066, PHY-0503729, PHY-0503629, PHY-0503641, PHY-0504224 and PHY-0705052), by the Department of Energy (Contracts DE-AC03-76SF00098, DE-FG02-91ER40688, DE-FG03-90ER40569, and DE-FG03-91ER40618), by the Swiss National Foundation (SNF Grant No. 20-118119), and by NSERC Canada (Grant SAPIN 341314-07).

\section{References}

[1] D. Clowe, M. Bradač, A. H. Gonzalez, M. Markevitch, S. W. Randall, C. Jones, and D. Zaritsky, Astrophysical Journal 648, L109-L113 (2006).

[2] D. N. Spergel, et al., Astrophysical Journal Supplement 170, 377-408 (2007).

[3] G. Jungman, M. Kamionkowski, and K. Griest, Physics Reports 267, 195 (1996).

[4] J. D. Lewin, and P. F. Smith, Astroparticle Physics 6, 87 (1996).

[5] D. S. Akerib, et al., Physical Review D 72, 052009-+ (2005).

[6] K. D. Irwin, S. W. Nam, B. Cabrera, B. Chugg, and B. A. Young, Review of Scientific Instruments 66, $5322(1995)$.

[7] K. D. Irwin, Applied Physics Letters 66, 1998 (1995).

[8] T. Shutt, B. Ellman, P. D. Barnes, A. Cummings, A. da Silva, J. Emes, Y. Giraud-Heraud, E. E. Haller, A. E. Lange, and R. R. Ross, Physical Review Letters (ISSN 0031-9007) 69, 3425 (1992).

[9] Z. Ahmed, et al., Science 327, 1619 (2010).

[10] Z. Ahmed, et al., Physical Review D 81, 42002 (2010).

[11] Z. Ahmed, et al., Physical Review Letters 103, 141802 (2009)

[12] Z. Ahmed, et al., THE THIRTEENTH INTERNATIONAL WORKSHOP ON LOW TEMPERATURE DETECTORS-LTD13. AIP Conference Proceedings (2009) 1185, 659 (2009)

[13] M. Pyle, et al., THE THIRTEENTH INTERNATIONAL WORKSHOP ON LOW TEMPERATURE DETECTORS-LTD13. AIP Conference Proceedings (2009) 1185, 223 (2009) 